
cultures

Les cahiers de l'Acedle

15-3 | 2018

Quelles médiations en didactique des langues et des cultures?

\title{
Les langues et cultures d'origine vues par les enseignants au Portugal
}

Rosa Maria Faneca, Maria Helena Araújo e Sá et Sílvia Melo-Pfeifer

\section{(2) OpenEdition \\ Journals}

Édition électronique

URL : http://journals.openedition.org/rdlc/3727

DOI : $10.4000 /$ rdlc.3727

ISSN : $1958-5772$

Éditeur

ACEDLE

\section{Référence électronique}

Rosa Maria Faneca, Maria Helena Araújo e Sá et Sílvia Melo-Pfeifer, «Les langues et cultures d'origine vues par les enseignants au Portugal », Recherches en didactique des langues et des cultures [En ligne], 15-3 | 2018, mis en ligne le 01 septembre 2018, consulté le 20 avril 2019. URL : http:// journals.openedition.org/rdlc/3727; DOI : 10.4000/rdlc.3727

Ce document a été généré automatiquement le 20 avril 2019.

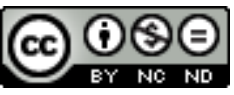

Recherches en didactique des langues et des cultures is licensed under a Creative Commons AttributionNonCommercial-NoDerivatives 4.0 International License 


\title{
Les langues et cultures d'origine vues par les enseignants au Portugal
}

\author{
Rosa Maria Faneca, Maria Helena Araújo e Sá et Sílvia Melo-Pfeifer
}

\section{Introduction}

1 Au cours des dernières décennies, le Portugal a reçu des immigrés issus de pays de différents continents (SEF - RIFA, 2016), rejoints plus tard par leur famille dont les enfants sont alors inscrits à l'école (DGEEC, 2016). En ce qui concerne la présence d'élèves étrangers dans les écoles portugaises, elle est évaluée à 49743 élèves (sur un total de 1641003 élèves) âgés entre 5 et 18 ans, dans l'enseignement primaire et secondaire, durant l'année scolaire 2014-2015 (DGEEC, 2016).

2 Cette arrivée de jeunes a changé la population scolaire portugaise, ce qui a contraint le système éducatif officiel à se confronter aux questions de diversité linguistique et culturelle. À partir de 2001, le ministère portugais a mis en place, en conséquence, des directives insérées dans la politique linguistique éducative, qui font référence à l'enseignement de la langue d'accueil, avec la création du portugais langue non maternelle (PLNM). Dorénavant, les écoles publiques garantissent l'enseignement de la langue d'accueil et un soutien pédagogique supplémentaire en LP.

3 Toutes les mesures publiées et implémentées dans le système éducatif concernent, presque exclusivement, la LP et l'utilité de son apprentissage ; elles s'avèrent néanmoins, de notre point de vue, insuffisantes, car la diversité linguistique et culturelle apporte d'autres défis, au-delà de la question de la norme scolaire et de la langue de scolarisation, à savoir la prise en compte de l'ensemble des ressources linguistiques et culturelles des apprenants en contexte pédagogique. Ce défi éducatif, qui implique de penser l'école comme un espace plurilingue et interculturel ou comme un lieu de développement individuel et social de la pluralité (Moore, 2006; Simon \& Maire Sandoz, 2008, p. 2), est assez exigeant pour les enseignants qui doivent se heurter, dans leurs pratiques, à des situations complexes marquées par la pluralité des langues, des cultures et des identités 
des apprenants. Dans ces circonstances, il existe une réelle nécessité de savoir comment les enseignants cernent la valeur pédagogique de la présence de la pluralité des langues et des cultures en général et, plus particulièrement, des LCO et de comprendre comment, dans un continuum qui peut aller de son ignorance totale à sa prise en compte systématique, ils gèrent les répertoires pluriels de leurs élèves. En même temps, il faut identifier les modèles conceptuels qui leur permettent d'encadrer ou de refuser les LCO en classe, les référentiels méthodologiques qui leur apportent des pistes pour des réponses contextualisées aux défis pédagogiques-didactiques et d'apprentissage.

\section{Contextualisation théorique}

\section{Langues d'origine : idéologies et statuts}

4 La notion de LCO est investie d'idéologies qui lui sont sous-jacentes (culturelles, démographiques, économiques, éducatives, historiques, politiques, religieuses, ethniques, sociolinguistiques, etc.). En fonction des groupes présents dans la société et en fonction des rôles qui lui sont assignés par la culture dominante ou par les propres locuteurs, l'étiquette LCO peut être problématique car, si pour certains élèves elle peut être un motif d'orgueil (Faneca, 2013, 2014), pour d'autres, elle peut être une raison de honte et de mépris (Castellotti, 2010; Nesteruk, 2010). Pour comprendre les statuts assignés aux langues présentes dans la société, il faut tenir compte de la place qu'occupent ces LCO dans l'imaginaire linguistique social, à savoir si elles sont valorisées et vues comme des atouts et/ou comme un capital linguistique par rapport aux autres LE enseignées dans le système scolaire portugais. Cette situation a été évoquée par de nombreux chercheurs, qui montrent que l'enseignement-apprentissage de plusieurs LE est perçu comme une plus-value et un capital linguistique pour la nation, pour le développement cognitif des individus et comme futur investissement professionnel (Cummins, 2005 ; Kondo-Brown, 2005 ; Lo-Phillip, 2010). Malheureusement, de nombreuses représentations dévalorisent l'enseignement-apprentissage, la transmission, la légitimation et le développement de la LCO (Cummins, 2005) au sein même des communautés transplantées. En amont, les systèmes scolaires prescrivent l'apprentissage des LE présentes dans les curricula sans tenir compte du bagage linguistique des élèves issus de l'immigration qui sont, pour la majorité, plurilingues car ils connaissent et parlent d'autres langues en dehors des LE (Faneca, Araújo e Sá \& Melo-Pfeifer, 2016). L'effet possible de ces idéologies linguistiques est que l'école transforme ces bi-plurilingues en monolingues (Cummins, 2005), empêchant un développement plurilingue durable. Il est vrai qu'au Portugal peu d'écoles publiques offrent la possibilité de s'engager dans un apprentissage formel dans la LCO (à l'exception du créole cap verdien, dans la périphérie de Lisbonne). Ainsi, la possibilité d'étudier la LCO est quasi inexistante (comme d'ailleurs dans de nombreux pays, le Portugal n'étant pas une exception). Il existe donc une réelle asymétrie entre l'enseignement-apprentissage de la LCO et l'enseignement-apprentissage de la LE (Beaudrie \& Ducar, 2005) et de la langue du pays d'accueil.

Dans ces conditions, s'il n'existe pas d'initiatives, de la part des communautés, pour créer des écoles de LCO pour ces jeunes, ces langues seront parlées difficilement par la génération suivante, contribuant ainsi à une disparition progressive de cette même langue, dévaluant les riches ressources culturelles et linguistiques de ces communautés (Faneca, 2013; Simões \& Melo-Pfeifer, 2010, pour des études menées au Portugal). Bien 
souvent lorsque ces sujets bi-plurilingues arrivent dans les écoles, la LCO se trouve dans un processus de passage de L1 à L2 (Lynch, 2002). De nombreuses études montrent que, pour un développement continu de la LCO, l'input des parents n'est pas suffisant : il faut idéalement se faire accompagner d'un input linguistique venant de la communauté, d'autres locuteurs, ainsi que d'un processus d'apprentissage (Nesteruk, 2010).

\section{Le locuteur de langues cultures d'origine : problèmes de définition et implications pédagogiques}

6 Les travaux concernant les LCO menés par de nombreux chercheurs sont importants dans la tentative de définition du concept des locuteurs de LCO et des implications pédagogiques qui en découlent (Carreira, 2004 ; Cummins, 2005 ; Fishman, 2001 ; Flores, 2015 ; García, 2005 ; Kondo-Brown, 2005 ; Little, 2010 ; Valdés, 2001 ; Van Deusen-Scholl, 2003; Wiley, 2001). Nous pouvons considérer deux types de définitions tenant compte de ces implications: une perspective basée sur la compétence linguistique du locuteur, défendue, entre autres, par Valdés (2001), affirmant que le locuteur de LCO est celui qui est éduqué à la maison dans une langue différente de la langue dominante, celle du pays d'accueil ; une deuxième définition, plus large, soutenue par Van Deusen-Scholl (2003), qui ne tient pas seulement compte de la compétence linguistique mais aussi de l'appartenance culturelle; les locuteurs de LCO sont ainsi définis comme un groupe hétérogène, qui comprend des natifs et des non-natifs, faisant parti de générations plus lointaines, mais qui se sentent culturellement liées à la langue, la parlant ou non, la revendiquant comme capital réel ou symbolique. Cette deuxième perspective peut soulever des polémiques parce qu'elle tient compte de locuteurs que l'on n'associe pas normalement aux communautés linguistiques et culturelles en cause. Une autre question qui se pose, est que, en contexte éducatif, en voulant créer des cours spécifiques de LCO, cette définition couvre autant de profils que l'on peut imaginer (Faneca, 2013; Ferreira, 2016). Ainsi, en positionnant la variété de locuteurs dans la même classe, comment l'enseignant va-t-il gérer cet enseignement-apprentissage? Pour ces raisons, de nombreux enseignants de LCO sélectionnent seulement les élèves qui déclarent avoir un degré de compétence linguistique, si minime soit-il à l'écrit ou à l'oral. Pour clarifier ces positionnements, beaucoup de tentatives sont faites pour trouver des contours plus définis, ce qui finira par conduire à un meilleur positionnement de ces locuteurs en cours de LCO et ainsi, à préparer des programmes plus adaptés à la diversité des profils (Fishman, 2001 ; Kondo-Brown, 2005 ; Wiley, 2001).

7 En bref, les compétences linguistiques, les liens culturels et le sentiment d'appartenance à une communauté particulière semblent être des facteurs importants pour la définition du profil d'apprenant de LCO, positionnant les locuteurs dans un «continuum de bilittéracies » (Hornberger, 2002). On remarque l'élasticité de la définition du locuteur de LCO, qui englobe des caractéristiques et des facteurs aussi bien culturels que linguistiques. Cependant, au moment où il est demandé aux enseignants de caractériser le profil de l'apprenant de LCO, ce sont les critères linguistiques liés à leur niveau de compétence qui sont davantage évoqués et non les culturels ou ceux concernant l'identité (Carreira, 2004). 


\section{Design méthodologique : questions de recherche, objectifs et méthodologie}

Cette étude s'insère dans une recherche plus vaste intitulée Le rôle des langues d'origine dans la compétence plurilingue des jeunes de minorités linguistiques et culturelles : une étude de cas dans les écoles du district d'Aveiro et de Coimbra, développée entre janvier 2013 et décembre 2015. La recherche vise à comprendre les relations que les élèves de minorités linguistiques et culturelles établissent avec les LCO et les autres langues de leur répertoire, et à analyser la mobilisation et la rentabilisation de ce répertoire plurilingue par les enseignants en salle de classe.

Pour atteindre les objectifs du projet, dans une première phase, un questionnaire a été proposé aux élèves qui fréquentaient deux écoles du centre du Portugal et 139 élèves ont accepté d'y répondre.

Dans une deuxième phase, nous avons mené des entretiens biographiques auprès de 20 élèves âgés de 13 à 18 ans, sélectionnés en tenant compte du contexte dans lequel s'est effectuée leur migration et de l'autorisation des parents pour participer aux entretiens.

Dans une troisième phase, celle que nous nous focalisons dans ce texte, nous avons distribué un questionnaire en ligne auprès des enseignants de deux écoles (collèges et lycées) du centre du Portugal qui ont accepté de participer à notre projet (une à Aveiro et une autre à Coimbra), au cours de l'année civile 2013/2014. Au total, 59 enseignants, de disciplines dites « linguistiques » et " non-linguistiques », ont accepté d'y répondre.

Cet article rend compte des résultats obtenus à partir du questionnaire et vise à cerner les représentations des enseignants quant à un travail pédagogique avec les LCO dans les écoles portugaises. L'hypothèse centrale de cette recherche, basée sur l'analyse des entretiens autobiographiques avec les élèves, en 2015 (phase 2), est que les enseignants semblent respecter la diversité linguistique et culturelle de ces élèves mais ne la prennent pas toujours en compte en salle de classe.

13 Ainsi, dans le cadre de ce texte, nous essaierons de répondre aux questions suivantes :

i. quelles sont les représentations des enseignants en ce qui concerne les LCO de leurs élèves ?

ii. de quelle manière la diversité linguistique et culturelle est-elle appréhendée et (dé)valorisée par les enseignants, notamment en termes cognitifs et affectifs?

iii. les enseignants prennent-ils en compte les langues des élèves allophones (et si oui, dans quelle mesure) dans l'apprentissage du portugais langue de scolarisation?

iv. Les enseignants déclarent-ils des besoins formatifs spécifiques pour aider à mieux gérer la diversité et le plurilinguisme?

\section{Présentation et discussion des résultats}

14 La contribution de Faneca, Araújo e Sá et Melo-Pfeifer (2015) a étudié la façon dont les jeunes de minorités linguistiques et culturelles se représentent en tant que sujets plurilingues et a montré que les langues dites d'origine (ou de première socialisation), aussi bien que les autres langues de leurs répertoires, sont importantes dans la constitution de leurs compétences plurilingues. Ces biographies ont montré que la LCO est, conjointement avec le portugais, un instrument de construction et d'affirmation des 
identités individuelles et collectives, ainsi qu'un outil de construction des relations interpersonnelles. Les élèves interrogés ont déclaré que les LCO leur ont permis de faire des transferts d'une langue à l'autre, d'une culture à l'autre, et qu'elles servent ainsi à établir et à consolider les repères linguistiques, culturels et identitaires. Pourtant, les entretiens avec ces élèves ont mis en évidence que l'école et les enseignants ne semblent pas toujours prendre en compte ni les langues des élèves ni leurs compétences plurilingues et pluriculturelles.

Suite à ces résultats, nous avons voulu présenter, dans cette étude, le point de vue des enseignants, et plus particulièrement, des 59 enseignants qui interagissent avec ces mêmes élèves.

\section{Profil des enseignants}

Des 59 enseignants ayant répondu au questionnaire, 78,43\% sont de sexe féminin, 19,61\% de sexe masculin (1,96\% n'a pas répondu). Leur moyenne d'âge est de 47 ans. La majorité est née au Portugal (4 sont nés au Brésil, 2 en France, 2 au Venezuela, 2 au Canada, 2 en Angola et 1 au Mozambique). 96,08\% d'entre eux se déclarent de nationalité portugaise ; $37,25 \%$ déclarent avoir également vécu dans un autre pays que le Portugal.

$78,43 \%$ d'entre eux possèdent une licence, $11,76 \%$ une maîtrise et 1,96 \% un doctorat. Au niveau professionnel, $90 \%$ des interviewés enseignent depuis plus de 15 ans, 7,84\% depuis une période se situant entre 10 à 15 ans. À peine 9,80\% d'entre eux sont titulaires du ministère de l'éducation et les autres sont contractuels. $41 \%$ d'entre eux enseignent des disciplines linguistiques (DL) ${ }^{1}$ et $49 \%$ des disciplines non linguistiques (DNL) $2.75,13 \%$ de ces enseignants enseignent au collège $\left(22,89 \% \text { en } 6^{\mathrm{e}}-5^{\mathrm{e}} \text { et } 52,24 \% \text { en } 4^{\mathrm{e}}-3^{\mathrm{e}}\right)^{3}, 34,25 \%$ enseignent au lycée et $29,11 \%$ enseignent en lycée professionnel les enseignements technologiques et professionnels, en vue de préparer un baccalauréat technologique ${ }^{4}$.

\section{Gestion pédagogique de la diversité linguistique et culturelle}

Dans cette section, il s'agit, d'une part, d'appréhender les connaissances que ces enseignants ont à l'égard des langues et des cultures de leurs élèves de minorités linguistiques et culturelles et, d'autre part, de comprendre si elles sont valorisées et mobilisées en contexte scolaire et dans la salle de classe. C'est ainsi que nous obtenons les résultats suivants :

Tableau 1 : Connaissances des enseignants concernant les LCO des élèves de minorités linguistiques et culturelles

\begin{tabular}{|l|l|l|l|}
\hline & Oui & Non & $\begin{array}{l}\text { Sans } \\
\text { rép. }\end{array}$ \\
\hline $\begin{array}{l}\text { 13 - Avez-vous des élèves de minorités linguistiques et culturelles } \\
\text { dans vos salles de classe? }\end{array}$ & $\mathbf{6 4 , 4 2} \%$ & $\mathbf{5 , 0 8} \%$ & $\mathbf{1 3 , 5 6 \%}$ \\
\hline $\begin{array}{l}14 \text { - Connaissez-vous la(es) langue(s) et cultures d'origine de vos } \\
\text { élèves de minorités linguistiques et culturelles? }\end{array}$ & $\mathbf{4 5 , 7 6 \%}$ & $\mathbf{3 7 , 2 9} \%$ & $\mathbf{1 6 , 9 5 \%}$ \\
\hline
\end{tabular}


Les résultats des questions 13 et 14 montrent que $64,42 \%$ de ces enseignants savent qu'ils ont des élèves de minorités linguistiques et culturelles dans leurs salles de classe et que $45,76 \%$ d'entre eux sont capables de les nommer.

Pour savoir quel rôle l'école attribue aux LCO, d'après ces enseignants, l'analyse des résultats obtenus indique que, pour $4,08 \%$, l'école considère la LCO très importante, pour $65,31 \%$, elle la considère comme importante et pour $30,61 \%$, elle ne la considère pas comme importante (tableau 2).

Tableau 2 : Importance accordée à la LCO

\begin{tabular}{|l|l|l|}
\hline \multicolumn{3}{|l|}{ Rôle attribué par l'école aux LCO } \\
\hline $\begin{array}{l}\text { Très important } \\
4,0 \%\end{array}$ & $\begin{array}{l}\text { Important } \\
65,3 \%\end{array}$ & $\begin{array}{l}\text { Pas important } \\
30,61 \%\end{array}$ \\
\hline DNL : 4,08 \% & DL : 12,09\% & DL : 20,40\% \\
& DNL : 53,20 \% & DNL : 10,21\% \\
\hline
\end{tabular}

Les enseignants des DNL semblent accorder plus d'importance aux LCO que les enseignants de DL. Les justifications données par ces enseignants se réfèrent surtout à leur potentiel pour mieux connaître les élèves et surtout pour mieux les aider en LP, comme nous pouvons le constater dans les extraits suivants, où les enseignants indiquent, essentiellement, les cours de portugais ou les activités visant à développer les compétences dans cette langue :

L'école se préoccupe des élèves provenant d'autres pays et organise des cours de soutien pour améliorer la langue portugaise. ID 48 (DNL)

En règle générale, l'école ne tient pas compte de la langue d'origine, elle se préoccupe, simplement, de savoir si l'élève a ou non besoin de soutien en portugais. ID 50 (DL)

Ces élèves fréquentent les cours de portugais langue non maternelle, d'autres ont des cours de soutien pour faciliter l'apprentissage de la langue portugaise et leur intégration scolaire et sociale, mais l'école n'est pas tenue d'offrir l'apprentissage de leurs LCO. ID 76 (DL)

Ces enseignants, même s'ils reconnaissent la diversité linguistique et culturelle et ses biens fondés, ne voient pas la présence des LCO, dans les contextes éducatifs, comme un potentiel pédagogique. L'apprentissage de la LP est considéré, par ces enseignants, comme la clé pour l'intégration de ces élèves et la seule ressource linguistique à capitaliser.

Pour comprendre si ces enseignants valorisent le capital linguistique et culturel des élèves de minorités linguistiques et culturelles, nous avons analysé les résultats obtenus dans une question liée au recours à la LCO de leurs élèves de minorités linguistiques et culturelles. Ceux-ci montrent que seuls 28,57\% affirment faire appel aux LCO de leurs élèves contre $69,39 \%$. Les enseignants qui ont répondu « oui » ( $46 \%$ de DL et $54 \%$ de DNL) soulignent qu'ils le font pour les raisons répertoriées dans le tableau 3.

Tableau 3 : Arguments des enseignants pour avoir recours à la LCO

\begin{tabular}{|l|l|l|}
\hline Arguments & Distribution & Total \\
\hline
\end{tabular}




\begin{tabular}{|l|l|l|}
\hline $\begin{array}{l}\text { Développement de compétences linguistico-communicatives en LP/ } \\
\text { Facilitateur d'apprentissage de la LP }\end{array}$ & $\begin{array}{l}\text { DL : 23,1 \% } \\
\text { DNL : 7,7 \% }\end{array}$ & $30,8 \%$ \\
\hline Comparaisons entre les langues & $\begin{array}{l}\text { DL : 23,1 \% } \\
\text { DNL : 7,7 \% }\end{array}$ & $30,8 \%$ \\
\hline Développement de connaissances de la diversité culturelle & DNL : 15,4 \% & $15,4 \%$ \\
\hline Stratégie de motivation & DNL : 7,7 \% & $7,7 \%$ \\
\hline $\begin{array}{l}\text { Stratégie de contrôle de la compréhension (p. ex., à travers le recours à la } \\
\text { traduction). }\end{array}$ & DNL : 7,7 \% & $7,7 \%$ \\
\hline Stratégie d'intégration & DNL : 7,7 \% & $7,7 \%$ \\
\hline
\end{tabular}

Nous n'avons pas constaté de grandes différences entre les arguments énoncés par les enseignants des DL ( $46 \%)$ et DNL (54\%), cette différence étant perçue en termes quantitatifs pour les deux premiers arguments («Développement de compétences linguistico-communicatives en LP/Facilitateur d'apprentissage de la LP» et «Comparaisons entre les langues ») qui sont davantage valorisés par les enseignants des DL. On peut néanmoins observer que les enseignants des DNL nomment une plus grande variété d'arguments pour recourir aux LCO : les quatre derniers arguments du tableau 5 ne sont signalés que par eux et dépassent les questions purement linguistiques pour embrasser des aspects liés, par exemple, à la motivation et aux savoirs concernant la diversité culturelle. Ces arguments mis en avant par les enseignants visent, en général, à aider les élèves à dépasser l'« obstacle » de la LP :

Pour aider l'élève dans la compréhension et l'acquisition de vocabulaire en langue portugaise. ID 68 (DNL)

Juste comme exemple et à titre comparatif, principalement quand surgissent des questions de fonctionnement de la langue. ID 32 (DL)

Comme stratégies au niveau de la compréhension, structure de la langue. ID 60 (DL)

Pour me servir des compétences déjà acquises pour améliorer l'apprentissage des autres langues. ID 60 (DL)

Les données recueillies révèlent que lorsqu'ils ont recours aux LCO, ils le font pour mieux intégrer ces jeunes et les aider à améliorer la LP, puisqu'ils considèrent sa maitrise comme le principal vecteur de réussite scolaire.

Parmi les 69,39\% des enseignants qui ont répondu qu'ils ne faisaient pas appel aux LCO, quelques-uns justifient leurs réponses avec les arguments présentés dans le tableau 4.

Tableau 4 : Arguments des enseignants pour ne pas avoir recours à la LCO

\begin{tabular}{|l|l|l|}
\hline Arguments & Distribution & Total \\
\hline Ce recours n'est pas ressenti comme nécessaire & $\begin{array}{l}\text { DL : } 10,3 \% \\
\text { DNL : } 13,7 \%\end{array}$ & $24 \%$ \\
\hline La diversité est grande et l'enseignant ne connait pas les LCO & $\begin{array}{l}\text { DL : 3,47 \% } \\
\text { DNL : 6,9 \% }\end{array}$ & $10,41 \%$ \\
\hline Inadéquation avec sa discipline & $\begin{array}{l}\text { DL : } 3,47 \% \\
\text { DNL : 6,9 \% }\end{array}$ & $10,41 \%$ \\
\hline
\end{tabular}




\begin{tabular}{|l|l|l|}
\hline Les élèves maitrisent le portugais & $\begin{array}{l}\text { DL : 3,48 \% } \\
\text { DNL : 5,22 \% }\end{array}$ & $8,70 \%$ \\
\hline Les élèves doivent maîtriser le portugais et non la LCO & DNL : 1,7 \% & $1,7 \%$ \\
\hline
\end{tabular}

Les résultats obtenus montrent que les enseignants des DNL valorisent et mobilisent moins le plurilinguisme et le pluriculturalisme de leurs élèves que les enseignants des DL. La tendance montre qu'ils n'y voient pas d'intérêt et que la présence/mobilisation explicite des LCO en salle de classe leur semble inadéquate dans le cadre de leurs disciplines. Ils reproduisent donc, dans leurs pratiques, les modèles de l'enseignementapprentissage monolingues. En général, les résultats de l'ensemble des répondants révèlent que la tradition monolingue continue à rendre difficile la valorisation et la mobilisation des LCO et le développement de pratiques plurilingues.

En ce qui concerne la valorisation des ressources linguistiques et culturelles préalables des élèves, seuls $22,45 \%$ de ces enseignants déclarent développer des activités liées à l'apprentissage de la LCO dans leurs cours mais ne les exemplifient pas, rendant impossible une vision plus concrète de leurs (éventuelles) pratiques.

L'acceptation de l'intégration des LCO en salle de classe, que ce soit ou non des cours de langues, semble encore poser problème pour 77,55 \% des répondants, d'un triple point de vue : i) la reconnaissance de leur utilité ; ii) l'articulation avec les contenus disciplinaires ; iii) le manque de préparation et de ressources éducatives.

Parmi les enseignants qui ont répondu à la question concernant l'intérêt que la pratique de ces activités culturelles, toutes liées aux traditions culturelles des pays en question, peut représenter chez les élèves portugais, $54,55 \%$ considèrent que la pratique de ces activités culturelles suscite beaucoup d'intérêt. Ces enseignants plus optimistes ne mettent pas en doute l'importance en soi de l'enseignement des LCO, celles-ci étant considérées comme un avantage potentiel pour le marché de l'emploi.

\section{Gestion des LCO des élèves de minorités linguistiques et culturelles et apprentissage du portugais}

Comme nous venons de le démontrer dans la section précédente, les enseignants disent valoriser les LCO de leurs élèves mais ne les voient pas comme un atout pédagogique. Pour eux, l'apprentissage de ces langues ne s'inscrit pas dans le cadre d'une politique linguistique cohérente et diversifiée mise en œuvre dans les orientations nationales des langues :

Ne répond pas aux programmes des langues enseignées. ID 42 (DL)

Les langues et les cultures d'origine ne sont pas intégrées dans les matières enseignées dans les écoles. L'école offre, seulement, le soutien en LP pour l'intégration de ces élèves dans le pays d'accueil. ID 58 (DNL).

Quant à la gestion de l'apprentissage, nous avons voulu comprendre quel rôle les enseignants attribuent à la LCO dans l'apprentissage du portugais. Les résultats obtenus montrent que $27,26 \%$ des enseignants considèrent que la LCO ne joue aucun rôle et qu'elle peut même avoir un effet négatif dans l'apprentissage du portugais, comme nous pouvons le constater dans les extraits suivants : 
Parfois, la langue d'origine nuit à l'apprentissage du portugais parce qu'à la maison la famille parle la langue d'origine et à l'école l'élève doit parler le portugais et cela exige beaucoup plus de ces élèves. ID 62 (DNL)

Parce que si les parents utilisent souvent la langue d'origine, cela peut pénaliser l'élève et ainsi, il n'améliore pas aussi bien l'apprentissage de la langue portugaise. ID 36 (DNL)

Les autres 63,63\% estiment que la LCO a un rôle important dans l'acquisition du portugais, comme nous pouvons le vérifier dans le tableau 5.

Tableau 5 : Importance accordée à la LCO dans l'apprentissage du portugais

\begin{tabular}{|l|l|l|}
\hline $\begin{array}{l}\text { Très importante } \\
9,08 \%\end{array}$ & $\begin{array}{l}\text { Importante } \\
63,63 \%\end{array}$ & $\begin{array}{l}\text { Pas importante } \\
27,26 \%\end{array}$ \\
\hline DL $: 4,54 \%$ & DL $: 21,21 \%$ & DL $: 13,63 \%$ \\
DNL $: 4,54 \%$ & DNL $: 42,42 \%$ & DNL $: 13,63 \%$ \\
\hline
\end{tabular}

Les enseignants des DNL, pour qui la LCO joue un rôle important (42,42 \%), affirment que le bagage linguistique de ces élèves est à prendre en considération dans l'acquisition du portugais, car la maîtrise de la langue d'enseignement est un facteur primordial de réussite scolaire et d'intégration. Cependant, ils n'explicitent jamais comment cette importance peut se concrétiser ou être mise en avant en cours :

Parce qu'elle restaure et renforce les liens et ses racines. ID 19 (DNL)

La langue exprime la connaissance qu'une personne a du monde. Chaque pays représente un monde, un univers lexical et culturel. La confrontation, la comparaison de deux univers linguistiques différents ou similaires peuvent contribuer à faciliter la compréhension en langue portugaise. ID 47 (DL)

En ce qui concerne la gestion de l'apprentissage, nous avons également voulu comprendre s'ils constataient des influences des LCO dans l'apprentissage du portugais. Quant aux influences des LCO sur l'usage et l'apprentissage du portugais, les résultats obtenus montrent que $38,63 \%$ des enseignants $-15,91 \%$ de DL et $22,72 \%$ de DNL - déclarent constater des influences de la LCO dans le portugais, contre $61,37 \%$ affirmant le contraire. Les influences de la LCO qu'ils observent en portugais sont: l'adoption de termes ou expressions et les influences transcodiques au niveau lexical, l'accentuation/la diction, la prononciation et l'orthographe, l'expression orale, le caractère encore réduit du vocabulaire et les difficultés à interpréter. Pour ces enseignants, ces influences sont toutes plutôt négatives car les LCO sont censées compromettre la «bonne maîtrise » du portugais, c'est-à-dire, du portugais normatif du Portugal. Ces influences sont « visibles » et " audibles », répondent les enseignants, au niveau de la prononciation, du vocabulaire, des structures linguistiques, des influences lexicales et phonétiques, de la structure discursive et des difficultés quant à l'interprétation de textes en portugais. Ces enseignants justifient les écarts entre ce qui se dit et ce qui est préconisé par le bon usage de la LP à travers les différences interlinguistiques, notamment aux niveaux de l'accord nominal, de l'utilisation des pronoms, des concordances des formes verbales, de la formation des temps et de la prononciation. Les plus grandes difficultés semblent appartenir au niveau interlinguistique, mais nos sujets se réfèrent aussi à des difficultés de nature intralinguistique et intervariationnelle, en raison des caractéristiques et 
spécificités de la LP en tant que langue pluricentrique (en raison des diverses variétés du portugais : européen, brésilien, africain).

Ainsi, dans les discours de ces enseignants, l'enseignement des LCO ne se présente ni comme un outil de pouvoir ou d'empowerment ni comme un facteur de succès académique car, ce qui est perçu comme valable, du moins en contexte scolaire portugais, ce sont les résultats académiques en LP. Selon leur perspective, les LCO sont plutôt un obstacle et donc dévalorisées/occultées.

Notons donc que les principales difficultés sont perçues au niveau linguistique et que les enseignants ne montrent pas (ou peu) d'ouverture pour comprendre les relations sociales et identitaires qui se construisent avec/à travers ces langues, l'enrichissement des profils linguistiques ou le rôle crucial que ces répertoires peuvent avoir dans le développement linguistico-communicatif, ou encore l'importance et les avantages de l'apprentissage des LCO. Les enseignants ne semblent pas reconnaitre le besoin d'adopter ni de nouvelles stratégies, ni des méthodologies plus adéquates pour intégrer ou cadrer la diversité linguistique et interculturelle en cours. Ils ne cherchent pas de solutions pour remédier aux difficultés que leurs élèves rencontrent dans la langue portugaise (dont, par exemple, l'adoption d'une pédagogie différenciée). On peut cerner une volonté restreinte de mettre en œuvre des situations d'apprentissages diversifiées, significatives et fécondes pour les élèves et leurs LCO.

\section{La formation des enseignants}

Quant aux besoins de formation pour gérer la diversité et le plurilinguisme, 88,37\% des enseignants répondent n'avoir jamais participé à des actions de formation sur le plurilinguisme, l'interculturel et les LCO, principalement parce qu'ils n'en voient pas la nécessité. Seuls $11,63 \%$ des enseignants, tous de DL, déclarent avoir suivi une formation sur le thème de la diversité, les principales raisons évoquées étant la pertinence et l'actualité de cette thématique, la prise en compte de la diversité linguistique et culturelle, l'approfondissement des connaissances et l'envie d'obtenir des réponses quant aux diverses interrogations que cette problématique suscite. De la totalité des interviewés, seuls 53,49\% (dont 30,23\% de DL et $23,26 \%$ de DNL) manifestent l'envie de suivre une formation sur la thématique du bilinguisme et du plurilinguisme, dont les raisons sont présentées dans le tableau 6.

Tableau 6 : Arguments énoncés par les enseignants qui expliquent la volonté de suivre une formation

\begin{tabular}{|l|l|l|}
\hline Arguments & Distribution & Total \\
\hline $\begin{array}{l}\text { Approfondir leurs connaissances sur le plurilinguisme, l'interculturel et } \\
\text { les LCO }\end{array}$ & $\begin{array}{l}\text { DL : } 11,6 \% \\
\text { DNL : } \%\end{array}$ & $18,6 \%$ \\
\hline Améliorer leurs pratiques éducatives & $\begin{array}{l}\text { DL : 4.6\% } \\
\text { DNL : 7 \% }\end{array}$ & $11,6 \%$ \\
\hline Thème actuel et enrichissant pour gérer l'altérité & $\begin{array}{l}\text { DL : 7\% } \\
\text { DNL : 4,6\% }\end{array}$ & $11,6 \%$ \\
\hline Enrichissement culturel & $\begin{array}{l}\text { DL : } 2,3 \% \\
\text { DNL : } 4,7 \%\end{array}$ & $7 \%$ \\
\hline
\end{tabular}




\begin{tabular}{|l|l|l|}
\hline Valoriser les connaissances et la diversité des élèves & DL : $2,3 \%$ & $2,3 \%$ \\
\hline Développer les connaissances professionnelles & DL : 2,3\% & $2,3 \%$ \\
\hline
\end{tabular}

Nous constatons que l'argument le plus cité par les enseignants, qui obtient $18,6 \%$, concerne la volonté d'approfondir leurs connaissances sur le plurilinguisme, l'interculturel et les LCO, comme nous pouvons le constater dans les extraits suivants :

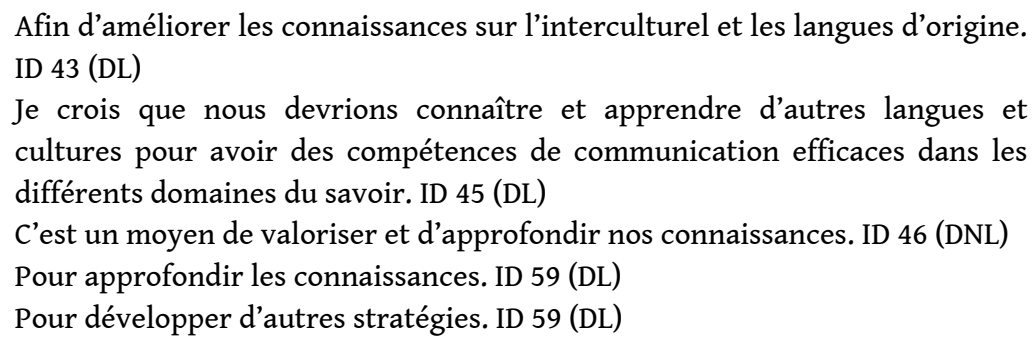

Pour s'insérer dans la société et réussir à l'école, les élèves de minorités linguistiques et culturelles ont besoin de développer certaines capacités, telles les capacités à se décentrer et à apprendre à connaitre et apprécier les différences linguistiques et culturelles de la société d'accueil (Banks, 2001). Par ailleurs, les enseignants sont censés promouvoir ces compétences chez leurs élèves. Pour cela, il est nécessaire qu'eux-mêmes laissent leur habitus monoculturel et monolingue derrière eux et accroissent leurs connaissances afin d'enseigner de manière efficace dans leurs nouveaux contextes. Nous appréhendons, grâce aux arguments énoncés par ces enseignants, que l'un des obstacles majeurs semble être la connaissance restreinte de la culture et de l'identité des élèves.

Quant à l'argument qui apparait en deuxième position, "Améliorer leurs pratiques éducatives ", qui obtient $11,60 \%$, plusieurs enseignants affirment être conscients de la diversité culturelle et linguistique qui existe aujourd'hui dans les écoles portugaises et qu'ils souhaitent se préparer, se munir de stratégies pour faire face aux besoins spécifiques que peuvent avoir les élèves et, ainsi, améliorer leurs pratiques éducatives :

Tout ce que je peux apprendre pour améliorer l'apprentissage de mes élèves sera important. ID 17 (DNL)

Pour trouver des réponses qui pourraient aider dans les méthodes de la classe. ID 47 (DL)

Parce qu'il y a sûrement des stratégies ou des méthodologies connues qu'on pourrait appliquer. ID 50 (DL)

Pour améliorer ma pratique éducative car nous avons de plus en plus d'élèves de différentes cultures. ID 68 (DNL)

Ces actions de formation pourraient m'aider à intégrer les langues et les cultures d'origine des élèves au niveau de l'apprentissage de ma discipline. ID 76 (DL)

Nos données montrent que ces acteurs ne sont que peu ou pas du tout préparés pour une prise en compte de la diversité linguistique et culturelle de leurs élèves pour qui la langue de l'école n'est pas toujours la langue parlée en famille. La formation pourrait les préparer à développer la capacité à comprendre l'élève dans ses différences et à gérer efficacement l'hétérogénéité dans les classes. Force est de constater, encore une fois, que les réponses révèlent surtout des lieux-communs, sont peu concrètes et contextualisées, et relèvent d'un certain flou pédagogique et didactique. 
argument, "Thème actuel et enrichissant pour gérer l'altérité ", obtient $11,6 \%$. Nous avons relevé, dans ce groupe de réponses, que ces enseignants considèrent le thème pertinent, actuel et enrichissant pour gérer l'altérité :

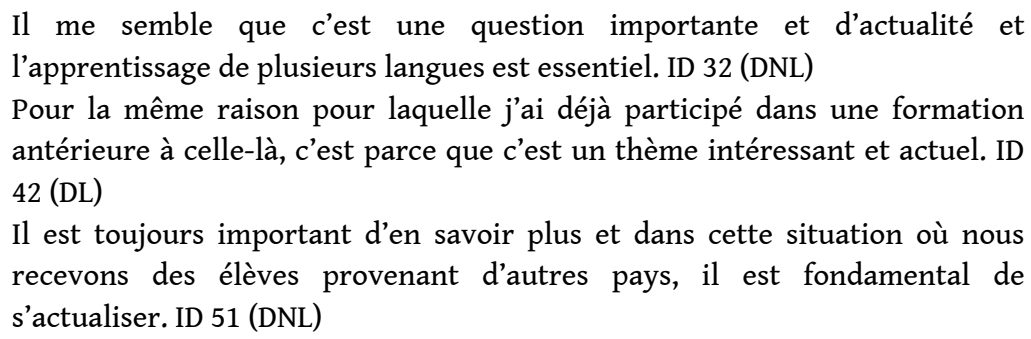

Ces enseignants expriment des attitudes ouvertes et accueillantes envers la diversité linguistique et culturelle qu'ils rencontrent dans leurs classes. Ils semblent être conscients de la richesse et de la valeur des diverses langues, mais la généralisation des réponses met en évidence le manque d'une réflexivité poussée par les situations vécues. enseignants reflètent une attitude positive face aux cultures des élèves et à l'importance de leurs apports, comme en témoignent les propos suivants :

Tout ce que je peux apprendre pour améliorer les apprentissages de mes élèves sera important. ID 17 (DNL)

Ces actions de formations pourraient m'aider à intégrer les langues et les cultures d'origine de mes élèves dans les apprentissages de ma discipline. ID 76 (DL)

Concernant les deux dernières typologies d'arguments, «Valoriser les connaissances et la diversité des élèves» et "Développer les connaissances professionnelles", les témoignages reconnaissent le besoin de développer une conscience plus critique, ainsi que la possibilité de s'engager dans la prise en compte de la diversité linguistique et culturelle. Cet engagement pourrait se faire, mais à l'aide de la formation, comme en témoignent les arguments suivants :

Une formation peut être importante pour l'enrichissement professionnel. ID 26 (DL)

Une formation peut m'aider à valoriser, à sa juste valeur, les connaissances des élèves concernés. ID 30 (DL)

Les résultats de notre étude montrent tout d'abord une certaine incapacité à exprimer des besoins de formation : les réponses révèlent plutôt des généralisations et très peu de rapprochement au vécu contextualisé et réfléchi. Ce constat nous amène à conclure qu'il faut renforcer l'offre formative et repenser les objectifs et les modalités d'une éducation à /par la diversité pour tous (Castellotti \& Moore, 2010). Cette formation, de notre point de vue, devrait :

i. les aider à développer un esprit critique face à la reconnaissance de la pluralité, de la diversité et de l'hétérogénéité, inter- et intra- linguistique ;

ii. indiquer aux enseignants des stratégies permettant d'optimiser le potentiel des élèves plurilingues ;

iii. les munir d'outils et de connaissances, tout en les encourageant à réfléchir sur les questions d'équité, d'identité et de diversité.

Recherches en didactique des langues et des cultures, 15-3 | 2018 


\section{Discussion} enseignants sont souvent peu préparés à gérer les variétés de langue et la variation dans les diverses formes qu'elle peut présenter dans les contextes scolaires, y compris l'hétérogénéité des profils linguistiques des élèves souvent socialisés et alphabétisés dans d'autres variétés de la LP et des tensions existantes entre ces normes linguistiques. Ces 
autres variétés ne sont pas toujours légitimées dans le contexte scolaire portugais car seule la variété du portugais européen (PE) est considérée comme un objet scolaire, d'apprentissage et soumis à évaluation.

Face à cette réalité, la diversité, en général, et la présence des LCO, en particulier, sont encore évaluées comme insignifiantes ou, plus gravement, comme des obstacles en salle de classe (Little, 2010). Au Portugal, les objectifs des structures et des politiques mises en place, plutôt que de s'orienter vers le développement du bi- et plurilinguisme et de la conservation des LCO, s'orientent plutôt vers la "portugalisation » des enfants issus de minorités linguistiques et culturelles. En effet, l'idéologie universaliste « à la portugaise ", même si elle oscille entre une politique d'intégration (ACIDI, 2010 ; PNAI, 2008 ; PNUD, 2009; Reis, 2016), voire d'assimilation, et une volonté de promouvoir une certaine pluralité linguistique et culturelle, reste profondément ancrée dans son habitus monolingue (Gogolin, 2008 ; Hélot, 2007).

\section{Conclusions et implications}

Les résultats obtenus dans ce contexte particulier et à travers une étude de nature compréhensive et descriptive, ne sont valables que pour l'échantillon enquêté et ne sont donc pas généralisables. Néanmoins ils permettent de décrire et d'améliorer la compréhension du thème en débat. Ainsi, à la lumière de nos résultats et dans le cadre d'une didactique de langues penchée vers une éducation plurielle (Alarcão et al., 2009; Kervran, 2012), il est nécessaire de repenser la formation des enseignants ainsi que de nouveaux matériels pédagogiques, de façon à inclure une "didactique de la pluralité " dans leurs discours et parcours professionnels. Ceci pourrait contribuer au développement de compétences pédagogiques, didactiques et de recherche et permettrait ainsi à l'enseignant, à partir d'une analyse réflexive de ses pratiques et de ses contextes de travail, de comprendre le concept de LCO dans sa différenciation, de l'articuler avec d'autres concepts et de comprendre les implications psycholinguistiques et didactiques qui découlent de la reconnaissance de la spécificité des locuteurs plurilingues.

Préparer des enseignants compétents qui savent comment répondre aux défis d'une école en mutation permanente exige, avant tout, du matériel d'apprentissage adapté et un profond changement dans les objectifs de formation des enseignants (Andrade, Araújo e Sá, et al., 2014). Ce changement devrait aider l'enseignant à développer une conscience critique et à construire une nouvelle identité professionnelle (Alarcão \& Araújo e Sá, 2010) capable de répondre de façon adéquate aux questions de la diversité, aux défis posés par la présence des LCO et à la spécificité des locuteurs plurilingues.

L'accès à l'imaginaire linguistico-professionnel des enseignants nous a permis de réfléchir aux configurations nécessaires au niveau du curricula et des scénarios de formation face aux défis posés par l'école, à savoir : faire face à la diversité linguistique et culturelle et préparer les enseignants à la gestion de cette diversité. En effet, ces résultats révèlent, de la part des enseignants, une certaine incapacité à gérer la complexité présente en salle de classe. Cette étude montre qu'il est important de continuer à travailler sur/avec les représentations des enseignants, de façon à comprendre les répercussions que certains positionnements peuvent avoir sur leurs actions. Cette étude a encore permis de comprendre comment les enseignants perçoivent la pluralité et, ainsi, de penser des réajustements de la formation initiale et continue pour améliorer leur travail, leurs représentations et leurs pratiques de classe. Une telle formation des enseignants viserait 
la construction de réponses situées, réactives et appropriées (Sá-Chaves, 2002) face à la diversité linguistique et culturelle. Cette formation pour la diversité devrait contempler une dimension méta-réflexive, de façon à permettre aux formés une meilleure connaissance de leurs représentations par rapport à la diversité, une réflexion qui conduirait à l'auto-connaissance sur et avec les autres, apportant des changements d'attitudes. Cette dimension réflexive devrait encore porter sur l'acte pédagogique, sur les contextes et enfin sur l'objet de l'enseignement-apprentissage des LCO (Valdès, 2001).

Le manque de formation des enseignants et le manque de politiques linguistiques éducatives en faveur du plurilinguisme affectent, forcément, la façon dont ces élèves sont intégrés dans les écoles, puisque nous vérifions qu'il existe peu de dialogue entre LCO et LP (et entre les LE présentes dans le curriculum). Compte tenu de ces défis, les enseignants ont besoin de matériels didactiques et de formations pédagogiques pour leur permettre de gérer et vivre l'altérité, et pour les aider à trouver des stratégies et des modèles éducatifs novateurs qui favorisent non seulement l'acceptation de la diversité linguistique et culturelle des élèves, mais surtout son intégration et prise en compte affective et cognitive (Beacco et al., 2010 ; Menken \& Garcia, 2010). Ils ont donc besoin de développer des compétences professionnelles pour agir pédagogiquement au sein d'une société en constante transformation (Schleicher, 2012).

Promouvoir une éducation plurilingue et interculturelle devient un enjeu nécessaire afin d'inclure toutes les compétences et tous les besoins linguistiques des différents groupes d'apprenants, œuvrant, de ce fait, à une éducation véritablement démocratique, inclusive et durable.

\section{BIBLIOGRAPHIE}

Alarcão, I. \& Araújo e Sá, M.H. (2010). Era uma vez... A didática de línguas em Portugal : Enredos, actores e cenários de construção do conhecimento. Cadernos do LALE, Série Reflexões 3. Aveiro : Universidade de Aveiro.

Andrade, A. I., Araújo e Sá, M. H., Faneca, R. M., Martins, F., Pinho, A. S., \& Simões, A. R. (2014). A diversidade linguística nos discursos e nas práticas de educação e formação. Aveiro : Universidade de Aveiro.

Alto Comissariado para a Imigração e o Diálogo Intercultural, I.P. (2010). Plano para a integração dos imigrantes (2010-2013), Lisboa : ACIDI.

Banks, J. A. (2001). “Citizenship Education and Diversity”. Journal of Teacher Education, nº 52, pp. 5-16.

Bardin, L. (2014). Análise de Conteúdo. (5.ํe ed.). Lisboa : Edições 70.

Beaudrie, S., \& Ducar, C. (2005). "Beginning level university heritage programs: Creating a space for all heritage language learners". Heritage Language Journal, no 3. pp. 1-26. 
Beacco J.-C., Byram, M., Cavalli, M., Coste, D., Cuenat, M. E., Goullier, F. et al. (2010). Guide pour le développement et la mise en œuvre de curriculums pour une éducation plurilingue et interculturelle. Strasbourg : Conseil de l'Europe.

Castellotti, V. (2010). « Les enseignements de « langues (et cultures) d'origine ». Chronique d'une disparition opportune ?» In Cadet, L. Goes, J., \& Mangiante, J.-M. (dir.), Langue et Intégration. Dimensions institutionnelle, socio-professionnelle et universitaire. Bruxelles : Peter Lang. pp. 83-94.

Castellotti, V. \& Moore, D. (2010). « Valoriser, mobiliser et développer les répertoires plurilingues et pluriculturels pour une meilleure intégration scolaire ». In L'intégration linguistique et éducative des enfants et des adolescents issus de l'immigration études et ressources $n^{\circ} 4$. Conseil de l'Europe. https://www.coe.int/t/dg4/linguistic/Source/ Source2010_ForumGeneva/4ValoriserCastellottiMoore_FR.pdf.

Carreira, M. (2004). "Seeking explanatory adequacy: A dual approach to understanding the term 'heritage language learner”'. Heritage Language Journal, no 2. pp. 1-25.

Cummins, J. (2005). “A proposal for action: strategies for recognizing heritage language competence as a learning resource within the mainstream classroom". The Modern Language Journal, vol. 89, nº 4. pp. 585-592.

Direção-Geral de Estatísticas da Educação e Ciência, (2016). Educação em Números - Portugal 2016. Lisboa : Edição DGEEC. Disponible en ligne à http://www.dgeec.mec.pt/np4/17/.

Faneca, R. M. (2014). « Parcours du portugais langue d'origine en France et des langues d'origine au Portugal : regards croisés ». Synergies Portugal, no 2, pp. 41-52.

Faneca, R. M. (2013). “Aprendizagem e representações do português língua de herança por luso (fono)descendentes em França em contextos não-formais”. Revista Indagatio Didactica, vol. 5, ํo 3 . pp. 29-49. Disponible en ligne à http://revistas.ua.pt/index.php/ID.

Faneca, R. M., Araújo e Sá, M. H. \& Melo-Pfeifer, S. (2016). “Is there a place for heritage languages in the promotion of an intercultural and multilingual education in the Portuguese schools?" Language and Intercultural Communication, vol. 16, nำ. pp. 44-68.

Faneca, R. M., Araújo e Sá, M. H. \& Melo-Pfeifer, S. (2015). « Le rôle des langues d'origine dans la compétence plurilingue des jeunes de minorités linguistiques et culturelles : une étude de cas dans les écoles au Portugal ». Migrations Société : "Éducation à la diversité et langues immigrées : expériences européennes”, vol. 162, nํ27. pp. 101-120.

Ferreira, T. (2016). “Faço o Pino !” : Representações e Práticas de Professores de Português Língua de Herança. In Melo-Pfeifer, S. (coord.), Didática do Português Língua de Herança. Lisboa: LIDEL. pp. 245-274.

Fishman, J. (2001). “300-plus years of heritage language education in the United States”. In Peyton, J. K., Ranard, D. A. \& McGinnis, S. (dir.), Heritage languages in America: Preserving a national resource. Language in Education: Theory and Practice Washington, DC \& McHenry, IL: Center for Applied Linguistics \& Delta Systems. pp. 81-89.

Flores, C. (2015). "Understanding heritage language acquisition. Some contributions from the research on heritage speakers of European Portuguese”. Lingua, vol. 164/B. pp. 251-265.

García, O. (2005). “Positioning Heritage Languages in the United States”. Hispania, vol. 89, nº 4. pp. 601-605.

Gogolin, I. (2008). Der monolinguale Habitus der multilingualen Schule. Münster : Waxmann.

Hélot, C. (2007). Du bilinguisme en famille au plurilinguisme à l'école. Paris: l'Harmattan. 
Hornberger, N. (2002). "Multilingual Language Policies and the Continua of Biliteracy: An Ecological Approach". Language Policy, n⿳o 1. pp. 27-51.

Kervran, M. (2012). Vers une didactique inclusive des langues à l'école primaire. Eveil aux langues et approches plurielles : de la formation des enseignants aux pratiques de classe. Paris: L'Harmattan. pp. 31-39.

Kondo-Brown, K. (2005). “Differences in Language Skills: Heritage Language Learner Subgroups and Foreign Language Learners". The Modern Language Journal, vol. 89, nº 4. pp. 563-581.

Little, D. (2010). The linguistic and educational integration of children and adolescents from migrant backgrounds. Brussels : Council of Europe. Disponible en ligne à http://www.coe.int/t/dg4/ linguistic/Source/Source2010_ForumGeneva/MigrantChildrenConceptPaper_EN.pdf.

Lo-Philip, S. (2010). “Towards a theoretical framework of heritage language literacy and identity processes”. Linguistics and Education, no 21. pp. 282-297.

Lynch, A. (2002). "The Relationship between Second and Heritage Language Acquisition: Notes on Research and Theory Building”. Communication présentée dans le Professional Development of Heritage Language Teachers. In Second National Conference on Heritage Languages in America, 19 octobre 2002, Tyson's Corner, VA, EUA.

Menken, K. \& García, O. (dir.). (2010). Negociating language policies in schools: Educators as polymakers. Nova Iorque : Routledge. pp. 1-9.

Moore, D. (2006). Plurilinguismes et école. Paris : Didier.

Nesteruk, O. (2010). "Heritage language maintenance and loss among the children of Eastern European immigrants in the USA". Journal of Multilingual and Multicultural Development, vol. 31, no 3 . pp. 271-286.

PNAI (2006-2008). “Plano nacional para a inclusão”. In Gabinete de Estratégia e planeamento (GEP). Disponible en ligne à http://www.gep.msess.gov.pt/estudos/peis/pnai0608_pt.pdf.

PNUD (2009). Rapport mondial sur le développement humain. Lever les barrières: Mobilité et développement humains. Disponible en ligne à http://hdr.undp.org/sites/default/files/ hdr_2009_fr_complete.pdf.

Reis, C. \& Gomes, N. (Coord.). (2016). "Indicadores de Integração de Imigrantes”. Relatório Estatístico Anual 2016. Lisboa : Alto Comissariado para as Migrações (ACM, IP).

Sá-Chaves, I. (2002). A construção de conhecimento pela Análise Reflexiva da Praxis. Lisboa: Fundação Calouste Gulbenkian/FCT.

Schleicher, A. (Ed.). (2012). Preparing teachers and developing school leaders for the 21st century: lessons from around the world. OECD Publishing. Disponible en ligne à http://doi.org/10.1787/9789264.

Serviço de Estrangeiros e Fronteiras (2016). Relatório de imigração, fronteiras e asilo. Oeiras : SEF. Disponible en ligne à https://sefstat.sef.pt/Docs/Rifa2016.pdf.

Simões, A. R. \& Melo-Pfeifer, S. (2010). « Les associations d'immigrants comme des espaces d'apprentissage de l'intégration : une étude comparative en contexte portugais ». In Cadet, L. Goes, J., \& Mangiante, J.-M. (dir.), Langue et Intégration - Dimensions institutionnelle, socioprofessionnelle et universitaire. Peter Lang. pp. 133-150.

Simon, D.-L. \& Maire Sandoz, M. O. (2008). « Faire vivre et développer le plurilinguisme à l'école : les biographies langagières au cœur de la construction d'identités plurielles et du lien social ». ELA. Études de linguistique appliquée, no151. pp. 265-276. 
Valdés, G. (2001). “Heritage languages students: Profiles and possibilities”. In Peyton, J. K., Ranard, D. A. \& McGinnis, S. (dir.), Heritage languages in America: Preserving a national resource Washington, DC \& McHenry, IL: Center for Applied Linguistics \& Delta Systems. pp. 37-77.

Van Deusen-Scholl, N. (2003). “Towards a Definition of Heritage Language: Sociopolitical and Pedagogical Considerations". Journal of Language, Identity, and Education, vol. 2, no 3. pp. 211-230.

Wiley, T. G. (2001). "On defining heritage languages and their speakers". In Peyton, J. K., Ranard, D. A. \& McGinnis, S. (dir.), Heritage languages in America: Preserving a national resource. Washington, DC \& McHenry, IL: Center for Applied Linguistics \& Delta Systems. pp. 29-36.

\section{NOTES}

1. Disciplines de portugais, latin, anglais, français, espagnol.

2. Disciplines de biologie/géologie, histoire, mathématiques, sciences naturelles, éducation physique, physique/chimie, arts visuels et arts plastiques, géographie, informatique, psychologie, éducation religieuse et morale.

3. Au Portugal, les 4 années du collège sont divisées en deux cycles, la $6^{\text {è }}$ et $5^{\text {è }}$ appartiennent au $2^{\text {è }}$ cycle et la $3^{\grave{e}}$ et $44^{\mathrm{è}}$ au $3^{\grave{e ̀ ~}}$ cycle du collège.

4. Comptabilité, administration, gestion, géométrie descriptive, technologies de l'information et de la communication.

5. ID : Identification de l'enseignant

\section{RÉSUMÉS}

Cet article présente les premiers résultats d'une vaste recherche réalisée en milieu scolaire portugais et dont les objectifs sont, d'une part, de comprendre la façon dont les enseignants, en milieu scolaire du centre du Portugal, facilitent ou non la mise en place de stratégies d'enseignement-apprentissage qui valorisent et reconnaissent la diversité linguistique et culturelle des élèves de minorités linguistiques et culturelles et, d'autre part, de savoir s'ils mobilisent les langues et cultures d'origine (LCO) dans leurs planifications, activités et discours en salle de classe.

This article presents a first set of results of a broader research carried out in Portuguese schools and whose objectives are on the one hand to understand the form as the teachers interviewed, teaching in schools in central Portugal, facilitate or not the establishment of teaching-learning strategies that value and recognize the linguistic and cultural diversity of students of linguistic and cultural minorities, and on the other hand whether they mobilize heritage languages and cultures (HLC) in their planning, activities and discourses in the classroom. 


\section{INDEX}

Keywords : heritage languages, teachers' representations, linguistic and cultural diversity, teacher education

Mots-clés : langues et cultures d'origine, représentations d'enseignants, diversité linguistique et culturelle, formation des enseignants

\section{AUTEURS}

\section{ROSA MARIA FANECA}

CIDTFF, Université d'Aveiro, Portugal

Rosa Maria Faneca est docteure en éducation - Didactique des Langues et chercheure de postdoctorat auprès du 'Centro de Investigação Didáctica e Tecnologia na Formação de Formadores' (Cidtff) de l'Université d'Aveiro, Portugal. Ses principaux centres d'intérêt de recherche sont la didactique des langues, didactique des langues d'origine, diversité linguistique et culturelle, répertoires plurilingues et pluriculturels et formation de professeurs.

rfaneca[at]ua.pt

LALE/CIDTFF, Departamento de Educação e Psicologia, Universidade de Aveiro, Campus de Santiago, 3810-193, Aveiro, Portugal

\section{MARIA HELENA ARAÚJO E SÁ}

CIDTFF/ Departamento de Educação e Psicologia, Université d'Aveiro, Portugal

Maria Helena Araújo e Sá est professeure associée à l'université d'Aveiro, Departamento de Educação e Psicologia et coordinatrice du 'Centro de Investigação Didáctica e Tecnologia na Formação de Formadores' (Cidtff) de l'Université d'Aveiro, Portugal.

helenasa[at]ua.pt

Departamento de Educação e Psicologia, Universidade de Aveiro, Campus de Santiago, 3810-193, Aveiro, Portugal

\section{SÍLVIA MELO-PFEIFER}

CIDTFF, Université de Hambourg, Allemagne

Sílvia Melo-Pfeifer est professeure à Université de Hambourg, Allemagne et chercheure auprès du 'Centro de Investigação Didáctica e Tecnologia na Formação de Formadores' (Cidtff) de l'Université d'Aveiro, Portugal.

silvia.melo-pfeifer[at]uni-hamburg.de

Fakultät für Erziehungswissenschaft, Universität Hamburg, Von-Melle-Park 8, 20146 Hamburg 